\title{
Single core configurations of saturated core fault current limiter performance of laboratory test models
}

\author{
Vittesh Naphade ${ }^{1}$, Vilas Ghate ${ }^{2}$, Gajanan Dhole ${ }^{3}$ \\ ${ }^{1,3}$ Department of Electrical Engineering, GES'sR.H.Sapat College of Engineering, Nashik, Maharashtra, India \\ ${ }^{2}$ Department of Electrical Engineering, Government College of Engineering, Amravati, Maharashtra, India
}

\begin{tabular}{l} 
Article Info \\
\hline Article history: \\
Received Jan 5, 2021 \\
Revised May 11, 2021 \\
Accepted Jun 1, 2021 \\
\hline
\end{tabular}

Keywords:

Current reduction

Distributed generation

Fault current limiter

Insertion voltage drop

Short circuit current

\begin{abstract}
Economic growth with industrialization and urbanization lead to an extensive increase in power demand. It forced the utilities to add power generating facilities to cause the necessary demand-generation balance. The bulk power generating stations, mostly interconnected, with the penetration of distributed generation result in an enormous rise in the fault level of power networks. It necessitates for electrical utilities to control the fault current so that the existing switchgear can continue its services without up-gradation or replacement for reliable supply. The deployment of fault current limiter (FCL) at the distribution and transmission networks has been under investigation as a potential solution to the problem. A saturated core fault current limiter (SCFCL) technology is a smart, scalable, efficient, reliable, and commercially viable option to manage fault levels in existing and future $\mathrm{MV} / \mathrm{HV}$ supply systems. This paper presents the comparative performance analysis of two single-core SCFCL topologies impressed with different core saturations. It has demonstrated that the single AC winding configuration needs more bias power for affecting the same current limiting performance with an acceptable steady-state voltage drop contribution. The fault state impedance has a transient nature, and the optimum bias selection is a critical design parameter in realizing the SCFCL applications.
\end{abstract}

This is an open access article under the CC BY-SA license.

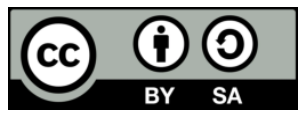

\section{Corresponding Author:}

Vittesh Naphade

Department of Electrical Engineering

Gokhale Education Society's R. H. Sapat College of Engineering, Management Studies and Research

Nashik, Maharashtra, 422 005, India

Email: vrnaphade@gmail.com

\section{INTRODUCTION}

The modern power systems across the world have undergone reforms with a tendency to separate the generation, transmission, and distribution entities. In an environment of decentralization, the utility handling the power network exercises no control over the locations as well as the scheduling of electricity generation. The dispersed generation is also the demand of the time as increased power demand can be met efficiently using the green energy resources coupled with the existing grid. The deployment of distributed generation (DG) can improve environmental pollution, defer the need for network expansions, reduce power losses, improve reliability and power quality [1]-[3]. The wind and solar generation are dominant renewable energy sources in the modern grids [4].

The power system networks, due to the coupling of these independent power producers, become very complex and the power system stability, as well as security, has become an issue of crucial importance. The modern power system stability and security can be augmented with rapid fault control and segregation [5]-[7]. However, modern power networks are spread over a wide area and exposed to environmental 
conditions and accidents, the chances of an unforeseen incident (faults) are inevitable. The fault effects are also then widespread and the high magnitude short circuit leads to severe thermal and mechanical stresses on the circuit components such as T \& D lines, transformers, and protective gears.

The applied protective gears, especially the circuit breaker, must be rated to handle the fault level and the higher the fault current the higher is the cost. The surging of mega power plants with an influx of the non-conventional dispersed generations particularly contributed to the high rise in fault level [8]. The fault currents approach or even exceed the limits of the short circuit handling capacity of the power network. The researchers, therefore, found considerable interest in the devices that can limit or control the ill effects of short circuit current [9], [10].

A fault current limiter is a device that can restrain extensive short circuit currents within the early instants of a fault. The successful implementation of the technology shall relieve the network hardware from the stresses. It leads to the longer service span of equipment with deferral of up-gradations or replacement. In the event of the new addition of the network, the application of FCL allows the use of equipment with lower specifications that may facilitate cost savings. The application of FCL in the network controls the contribution of subsystems to the short circuit problems. The reduction in fault current amplitudes also enables high system availability with parallel connection of generators and transformers, enhanced loadbearing capacity of subsystems, reduction of voltage sags and flicker, reduction of harmonics. [11]. The FCL application area lies in the bus couplers, incoming feeders, or the outgoing feeders.

The FCLs, based on whether the superconductor employed, are classified into two main technology groups as superconducting and non-superconducting as shown in Figure 1. The solid-state FCLs use power semiconductor switches and provide a very low impedance during steady-state operation with comparatively high power losses. However, these FCLs need ancillary electronics circuitry for fault detection and control, which results in delayed response and reaction to the fault [12]. Superconducting FCLs may be divided into two categories viz. quench type and non-quench type [13]. The quench type FCL relies on the transformation of the zero impedance state of a superconductor during steady-state operation to a significantly high impedance state during a fault condition. However, it also suffers from slow fault response and high recovery times, in addition to the costly superconductors with cryogenics for the application of these FCLs [14], [15]. The SCFCListhe non-quench type of FCLs.

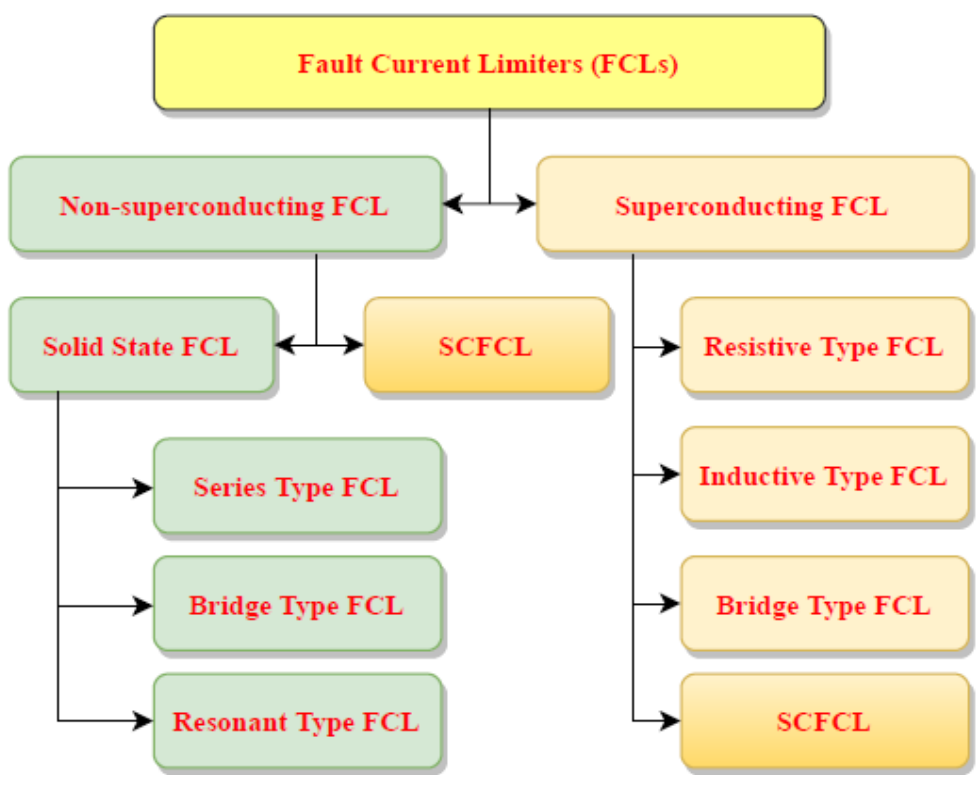

Figure 1. Classification of FCL technology

The SCFCL essentially uses the nonlinear magnetic characteristics of a core to realize a variable inductance. The limiting action, passively, can be realized without any external trigger circuit. The SCFCL technology shares similar electromagnetic principles of electrical devices as in transformers and power inductors, and hence it is a compatible introduction in the network. It is rugged in construction and offers immediate action and recovery with multi-shot ability. Since the SCFCL offers an inductive impedance during the fault, the fault energy remains stored in the magnetic field, and most of it returns to the system that 
enables efficient operation of the device. The SCFCL, being non-semiconductor based technology, is especially suitable for applications in medium and high voltage power distribution and transmission grids. It offers high impedance under transient conditions to enable the current limitation, and acts transparent to the system under steady-state. The instantaneous reaction and recovery, consecutive faults control, and high current clipping ability during transient operation (even for consecutive faults) are the strengths of the SCFCL. Although it possesses inherent advantages, the original double core configuration of SCFCL had a costly solution due to the substantial size and material requirements for its core and the windings. The ACDC coupling during the transient operation, large size, weight, volume, and cost have been challenges before commercialization of this topology. Recently, the advent of high-temperature superconductors regained the focus in the development of SCFCL and various small scale prototypes have been suggested in the literature [16]-[18]. The concept in its basic form [19] has seen many modifications and studies while following the developments. The improved characteristics of novel SCFCL can easily be tuned to the general and specific grid requirements by adjusting the physical parameters of the magnetic core structure, AC and DC winding parameters and by controlling the DC bias current [20], [21]. The work addressed here belongs to the nonsuperconducting, novel SCFCL.

In this paper, the two single-core novel SCFCL configurations have been examined experimentally and compared under similar operating environments. Since the SCFCL exploits the non-linear magnetic properties of the iron-core for affecting the current limiting action, the effect of changing the core magnetic states had investigated under four different DC bias levels, viz. 0A-3A with a step of 1A. The transient performance of the device during the faulted operation had also investigated.

\section{SCFCL: GENERAL PRINCIPLES AND WORKING}

The conventional dual-core configuration [19], [22] consist of three main parts, viz. magnetic cores, $\mathrm{DC}$ winding( $\mathrm{LV})$, and the $\mathrm{AC}$ windings $(\mathrm{HV})$, as shown in Figure 2. The iron cores have biased with a low voltage DC coil powered by a separate DC power source. It necessarily keeps them saturated during normal operations. For the clipping of each half-cycle of the current wave, two iron-cores have been used. In addition to full-cycle clipping, the induced emf (transformer action) at the DC coil during normal, steadystate operation is counter-balanced by the double core arrangement. The AC windings are connected differentially in series with the power line to be protected, producing anti-parallel magnetic fields.

During the steady-state operation, to force the iron cores in a deep saturation state, the DC bias current is kept injected in the DC coil. Therefore, it results in the low permeability of the magnetic core leading to very little impedance to the AC windings. The magnetic-core, in this case, works in the saturation region of the magnetization curve. During the faulted operation of the system, the limiting action has enabled to control of the short-circuit. In each half-cycle of the short-circuit current, high under the limiting state operation, the iron cores are, alternately, forced out of saturation. The operating point of the magnetic-core shifts in the linear (unsaturated) region of the B-H curve. Thus, a high inductance to the AC coil realized by the increased permeability controls the evolution of the short circuit current in the line. It is a passive triggering of the reactive impedance.

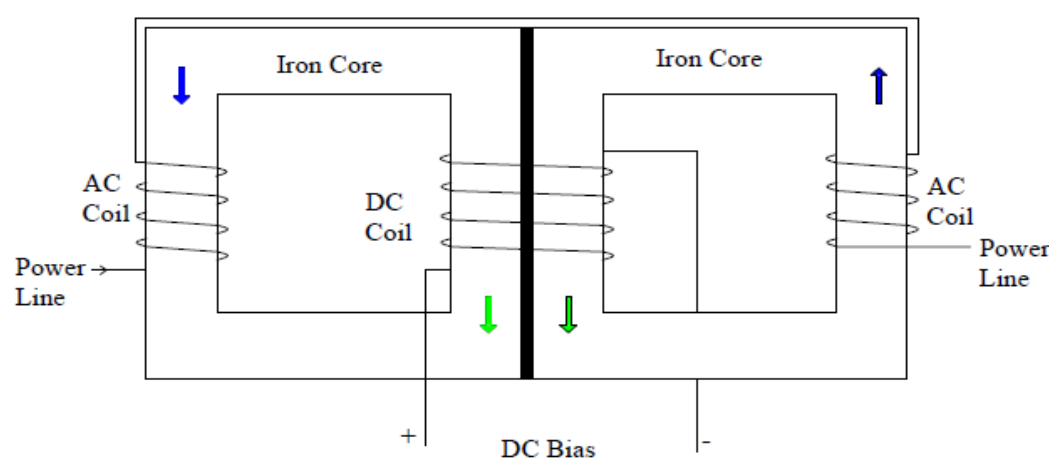

Figure 2. Dual core configuration of SCFCL

For the transperentsteady-state operation, the voltage drop, hence insertion impedance is required as minimum as possible. The necessary DC magnetizing force required to push the cores in the saturation during the non-limiting operation is given by, 


$$
N_{D C} I_{D C}=H_{D C} \times 2(2 w+2 h)
$$

where $\mathrm{N}_{\mathrm{DC}}$ : number(total) of DC turns, $\mathrm{I}_{\mathrm{DC}}$ : DC(bias) coil current, $\mathrm{H}_{\mathrm{DC}}$ : magnetic field intensity generated by the bias(DC) current, w: mean width of the core, and h: mean height of the core. In order to realize the transparent operation, in this case, the selected bias should be such that,

$$
\mathrm{H}_{\mathrm{DC}} \quad \mathrm{H}_{\mathrm{SAT}}
$$

where, $\mathrm{H}_{\mathrm{SAT}}$ is the magnetic field intensity at saturation of the iron-core. The impedance under the nonlimiting state, an insertion impedance, may be approximately calculated [23] as,

$$
Z_{i} \approx 2 \omega \frac{\mu_{0} N_{A C}^{2} A_{C}}{l_{A C}} \beta
$$

where, $\omega$ : angular frequency, $\mu_{0}$ : the permeability of the core, $\mathrm{N}_{\mathrm{AC}}$ : number of $\mathrm{AC}$ coil turns, $\mathrm{A}_{\mathrm{C}}$ : the cross sectional area, $1_{\mathrm{AC}}$ : the effective core length under $\mathrm{AC}$ coil(height of the $\mathrm{AC}$ coil), $\beta$ : the correction factor to account for the actual core geometry.

Under the fault (transient) condition, the magnetic cores are taken out of saturation alternately in each half-cycle, permeability increases resulting in high inductance to the coil that limits the fault current. In this case, the high magnitude short-circuits current results in comparatively large, de-magnetizing force at the core (alternatively), in each half cycle of the $\mathrm{AC}$ wave. For affecting the effective current limiting, the demagnetizing force is required [24] to be such that,

$$
\left(H_{D C}+H_{S A T}\right) l \geq N_{A C} \hat{I}_{F} \geq\left(H_{D C}-H_{S A T}\right) l
$$

where, $\ell:$ the mean length of the core, $\hat{I}_{F}$ : is the peak value of the short-circuit current. The iron cores, during the limiting state operation, alternately forced out of saturation. The de-saturated core offers high permeability resulting to high impedance for the affiliating AC coil, which dominates the total fault impedance realized by the device. The application of electromagnetic principles may be used to calculate the approximate fault impedance [23] given as,

$$
Z_{F} \approx 2 \omega \frac{N_{A C} A_{C} B_{S A T}}{\sqrt{2} I_{F}}
$$

where, $\mathrm{B}_{\mathrm{SAT}}$ : saturation flux density of the iron-core, and $\mathrm{I}_{\mathrm{F}}$ : the rms value of the limited short-circuit current.

The DC bias selection is very crucial for the desired SCFCL operation. If the bias applied to locate the operating point far away from the knee(on the right) of the magnetization curve, the magnetic state of even the differentiating fields (desaturating) core may still be in the saturation, and may not attend the high impedance faults, where the short-circuit currents are relatively lower. Whereas in the case of operating point location in the extra-shallow saturation region, the magnetic state of the same core(where the differential fields are acting) may lie on the linear region of the magnetization, which may lead to the high impedance state even during the non-limiting SCFCL operation. These two operating extremities demand the proper bias application that will ensure the acquirement of high impedance(lower currents) faults besides adequately low impedance(high transparency) running during the healthy state of the system.

\section{SINGLE CORE TOPOLOGIES}

Many feasible configurations, with the same principle of inherent impedance change, have been proposed in the literature. These configurations mainly differ in the bias designs, core shape, and AC circuit arrangements, aimed at dealing with the challenges in terms of large volume, cost, and the induced voltage in DC bias due to AC-DC coupling [25]. Furthermore, the use of superconductors has also been a barrier before commercialization. The following two single-core topologies, which can alleviate these issues, are the subject matter of the work presented in this paper. 


\subsection{Single AC winding model}

The novel topology, as shown in Figure 3(a), has a DC bias coil wound over one of the short legs of the strongly elongated magnetic core, and the AC coil over the complete core encircling both the longer legs. The design provides a closed magnetic path for the DC flux, and it acts as an open core for the AC flux. As the mean length of magnetic circuit has reduced, it can be forced to the saturated mode by moderate DC bias magnetizing force. The AC flux supports the DC flux in one of the legs and counteracts the DC flux in the other longer limb of the core. In steady-state operation, the magnetic core is driven to saturation by the DC bias. The steady-state AC load current is unable to cause desaturation of the iron-core resulting in the low inductance to the $\mathrm{AC}$ coils and transparent to the system. In a faulty case, a large current alternately desaturates the core segments carrying counteracting AC-DC flux. Hence, the high permeability leads to increased inductance to the AC coil initiating the limiting action. This design has the advantage of only one magnetic core and one AC coil for each phase. The novel design reduces iron-core volume, weight, cost, and enable it to be adaptable to the specific service requirements. Also, the orthogonal placement of the winding reduces the AC-DC coupling and consequent induced emf across the DC circuit [26].

\subsection{Double AC winding model}

The other modified design of the single iron-core configuration, as shown in Figure 3(b), has two DC bias coils and two AC coils. The basic principles of an open AC magnetic circuit and an orthogonal placement of AC and DC coils remain unchanged [21]. The two DC coils are wound over the shorter limbs while the two AC coils are on the long limbs. The DC flux travels through the complete core section and follows the closed magnetic path through the core. The AC coils are differentially coupled and resulting AC fluxes complete their path through air behaving like an open (AC) magnetic circuit. The AC fluxes reverse their direction with each alternate half cycle of the $\mathrm{AC}$ wave. The operating principle, as described in subsection 3.1, remains the same.

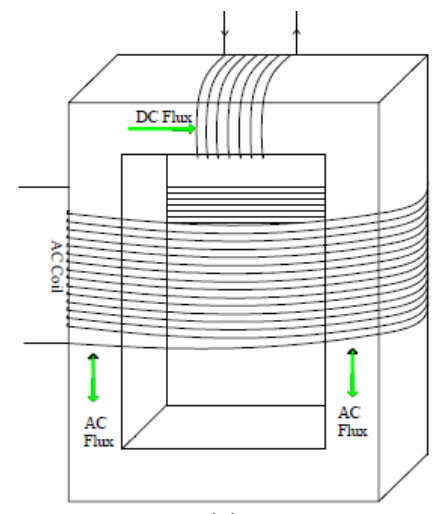

(a)

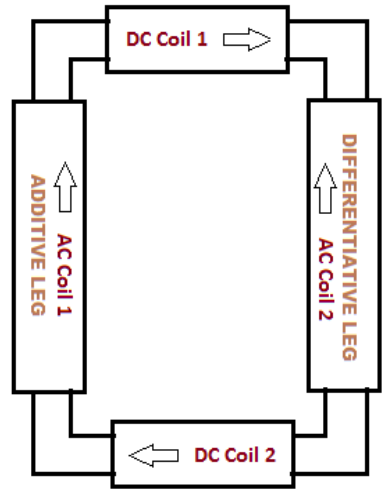

(b)

Figure 3. Single core topologies; (a) single AC winding model, (b) double AC winding model

\section{TEST SYSTEM DISCRIPTION}

The similar elongated cores of high-grade M4 electrical steel material have been used for the SCFCL models with the physical parameters as described in Table 1. A simple power system with a singlephase autotransformer $(0-250 \mathrm{~V})$ as a source and a lamp bank as a load (resistive) has been realized in the laboratory. A controlled DC source (0-10A) has biased the core. The worked-out digital logic has enabled the contactor to realize the controlled short circuit ( 7 cycles) across the load. The different faults are applied with the variations in the DC bias. A $50 \mathrm{MHz}$, dSpaceMicroLab box is used to capture the fault events data with a sampling frequency of $10 \mathrm{kHz}$. The faults at the operating voltage of $100 \mathrm{~V}$ (RMS) were executed across the load. The low voltage is selected looking at the safety of the wiring and pieces of equipment. The CT/PTs of appropriate specifications scaled-down the parameters for measurements in the simple power system. A picture of an actual experimental setup is shown in Figure 4(a).

Table 1. Physical parameters of the test models (core dimensions in $\mathrm{mm}$ )

\begin{tabular}{cccccccc}
\hline Leg width & Leg depth & Mean width & Mean height & $\begin{array}{c}\text { Area of } \\
\text { C.S. }\left(\mathrm{mm}^{2}\right)\end{array}$ & Mean Length & Turns DC(total) & Turns AC(total) \\
\hline 16 & 30 & 54 & 138 & 480 & 384 & 140 & 300 \\
\hline
\end{tabular}

Single core configurations of saturated core fault current limiter performance of .... (Vittesh Naphade) 
The two single-core configurations of the SCFCL have been implemented as laboratory test models. The two models use the same core material, dimensions, and the quantity of copper material employed for the $\mathrm{DC}$ and $\mathrm{AC}$ windings. The only difference lies in the arrangements of $\mathrm{DC}$ and $\mathrm{AC}$ winding as depicted in the Figure 3. The separate experiment is carried out to study the magnetic behaviour of an iron core, and the experimentally plotted B-H curve is shown in Figure 4(b). The B-H curve data is also fitted by least square approximations, and the analytical model is given by,

$$
\begin{aligned}
& B=a H^{b}+c \\
& \mu=\frac{d B}{d H}=a b H^{b-1}
\end{aligned}
$$

where the coefficients (using cftool in MATLAB) are given by $a=-4.452, b=-0.2067$ and $c=3.724$. As the current limiting performance is largely affected by the magnetic behavior of the iron-core, the characteristics of the iron-core were studied first. The analytically calculated DC bias of $1 \mathrm{~A}$ to force core in the saturation had validated from the plotted B-H curve. It leads the decision on the values of bias current, to note the effect of working magnetic states of the iron-core. The experimental findings on the current limiting performance are discussed in the next section.

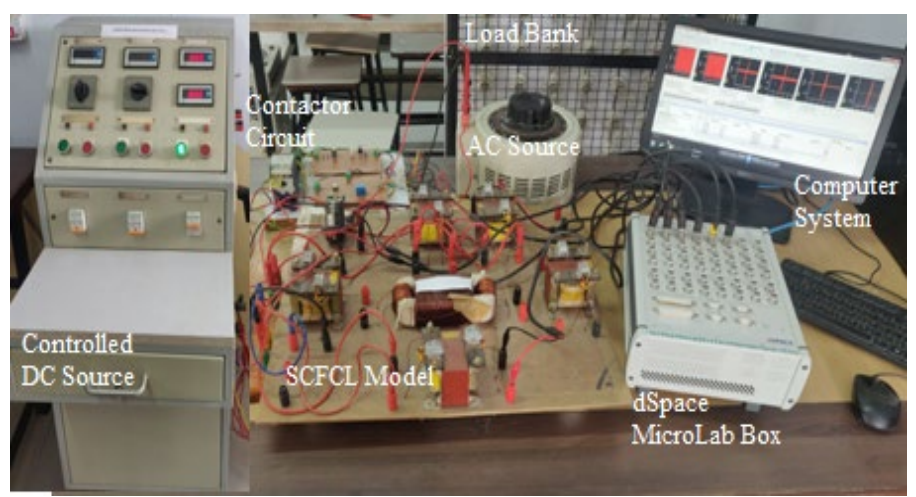

(a)

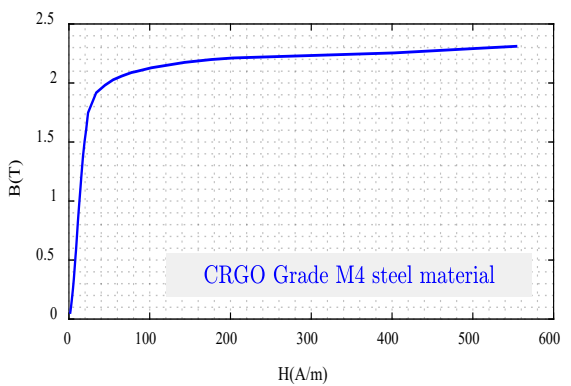

(b)

Figure 4. A picture of; (a) an experimental setup, (b) B-H curve for the core material

\section{RESULTS AND DISCUSSION}

\subsection{The current reduction and insertion drop}

Initially, the faults are realized without FCL in the circuit. The short circuit current (without FCL) has a maximum peak of 119A, the steady-state peak value of 111 , and the rms measured current of 77A. It has significantly reduced by the application of SCFCL models. Figure 5 shows the prospective short circuit current superimposed with the controlled current for the double and the single AC winding models.

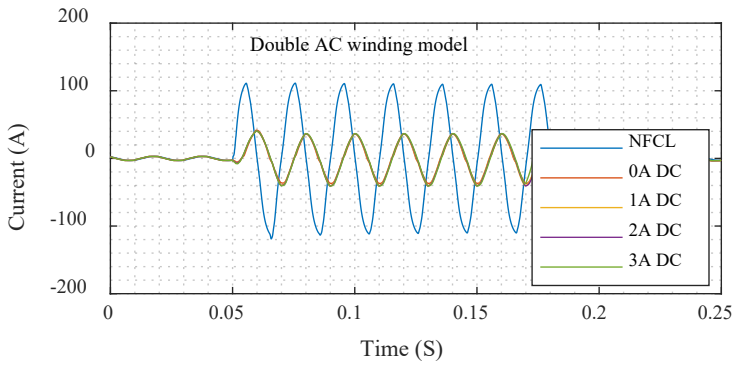

(a)

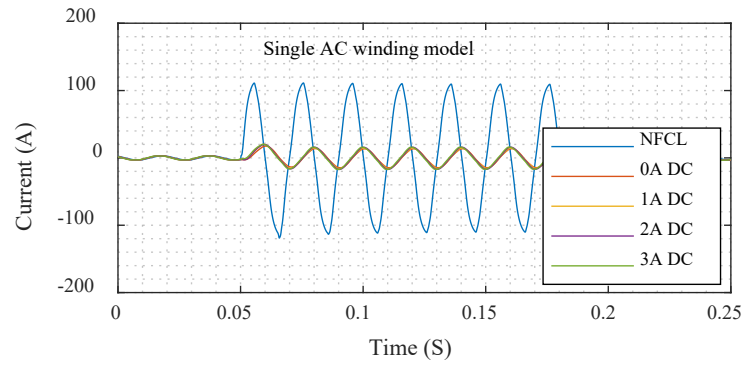

(b)

Figure 5. The prospective and limited currents; (a) double AC winding model, (b) single AC winding model 
The zoomed sections of the controlled currents are shown in Figure 6. It has been noticed that, in both cases, the controlled current peak magnitudes have marginally increased with the increasing bias. The quantitative representation of current reduction is expressed in terms of current reduction rates [10], as defined in (8)-(10),

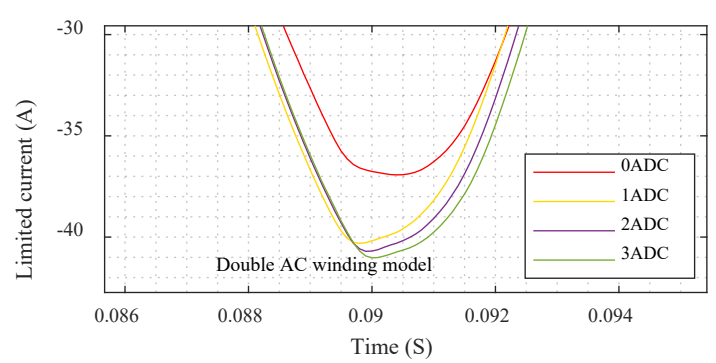

(a)

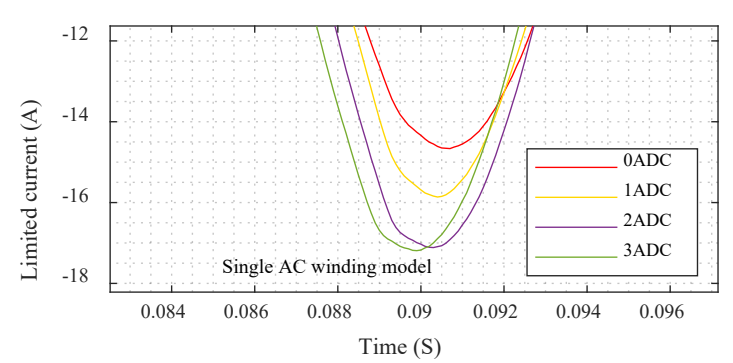

(b)

Figure 6. The zoomed limited currents; (a) double AC winding model, (b) single AC winding model

$$
\begin{aligned}
& \delta_{\text {max }}=\frac{\mathrm{I}_{\mathrm{p}_{\max }}-\mathrm{I}_{\mathrm{c}_{\text {max }}}}{\mathrm{I}_{\mathrm{max}}} \\
& \delta_{\mathrm{SS}}=\frac{\mathrm{Ip}_{\mathrm{SS}}-\mathrm{Ic}_{\mathrm{SS}}}{\mathrm{I}_{\mathrm{SS}}} \\
& \delta_{\mathrm{rms}}=\frac{\mathrm{Iprms}-\mathrm{Ic} \mathrm{rms}}{\mathrm{Iprms}}
\end{aligned}
$$

where, Ipmax, Ipss and Iprms are the maximum peak, the steady-state peak, and the rms current of prospective fault current and $\mathrm{Ic}_{\mathrm{max}}$, $\mathrm{Ic}_{\mathrm{SS}}$ and Icrms are that of controlled current respectively. The measured values of current reduction rates for the double AC winding model and the single AC winding model with the

\begin{tabular}{|c|c|c|c|c|}
\hline \multirow{2}{*}{$\begin{array}{c}\mathrm{DC} \\
\operatorname{bias}(\mathrm{A})\end{array}$} & \multicolumn{3}{|c|}{$\%$ Current reduction rates } & \multirow{2}{*}{$\begin{array}{c}\% \text { voltage } \\
\text { drop }\end{array}$} \\
\hline & max-peak & steady-state peak & rms & \\
\hline \multicolumn{5}{|c|}{ Double AC winding model } \\
\hline 0 & 66.1 & 66.65 & 68.04 & 11.49 \\
\hline 1 & 65.81 & 63.73 & 66.45 & 4.31 \\
\hline 2 & 65.54 & 63.38 & 66.11 & 3.56 \\
\hline 3 & 64.93 & 63.08 & 65.7 & 3.08 \\
\hline \multicolumn{5}{|c|}{ Single AC winding model } \\
\hline 0 & 85.27 & 86.77 & 87.25 & 18.22 \\
\hline 1 & 83.18 & 85.78 & 86.76 & 15.81 \\
\hline 2 & 83.12 & 84.66 & 85.6 & 12.50 \\
\hline 3 & 83 & 84.56 & 85.49 & 12.38 \\
\hline
\end{tabular}
voltage drop contributions in the system are mentioned in Table 2.

Table 2. The current reduction rates and the insertion drops

The first peak, the steady-state, and the rms current reduction rates, in both cases of the models, were found to be decreasing with the increasing level of the DC bias. It has been minimum for maximum applied bias and vice-versa. It is because the increase in bias level shifts the operating point towards the right on the magnetization curve. The net demagnetizing force, a resultant of DC and counteracting AC field, then available to bring the core operation towards linearity reduces. While the operating point progresses towards the right, the value of the working permeability decreases, and the resulting impedance offered by the device decreases.

The unbiased core operation (DC bias of $0 \mathrm{~A}$ ) has shown maximum current reduction at the cost of objectionable voltage drop under pre-fault operation (non-limiting state) for both the double and single AC winding models. The substantial voltage drop contributions, in this case, reveals the linear region (below the 
knee point) operation of the device under its nonlimiting state. Since the permeability of the core is high in this region, it results in a high impedance to the AC coils and subsequent higher voltage drop. The maximum, and discouraging values of voltage drop (0A bias) for both the models expose the normal-reactor operation of the device. It is an impractical operating condition, and against the working principle of the device, however, useful to compare the performance with the other cases of bias.

The current reduction rates in the case of the double AC winding model are marginally varying from the bias level of $1 \mathrm{~A}$. Also, the percent voltage drop under normal conditions (insertion voltage drop) does not vary remarkably with the higher levels of bias. For the double AC winding model, the operation at $1 \mathrm{ADC}$ has, therefore, confirmed the core saturation under normal operation where the voltage drop is acclaimed as $4.31 \%$, a significant change from $11.49 \%$ at $0 \mathrm{~A}$. For all the other higher biasing, the voltage drop is, also, seen to be reducing insignificantly at the cost of minor curtailments in current clipping. This demonstrates the excess excitation of the core does not turn out significant performance improvement, and the optimum selection of bias is an influential consideration while designing the SCFCL system.

In the case of the single AC winding model, though the current reduction rates are acclaimed significantly on the higher side, the insertion drops are unacceptably high, more than $12 \%$ in all the cases of the bias. This demonstrates the shallow saturation region (around the knee point region) operation of the core even beyond the bias of $1 \mathrm{ADC}$. The graphical comparison of insertion drops for both models is shown in Figure 7. The single AC winding model hence appeals more bias compared with the double AC winding model for affecting the identical performance. As bias level increases, the insertion drop in the single AC winding model also decreases, indicating shifting of operating point towards the nonlinear region of the magnetization curve. Since the current reduction is to be maximized and insertion drop to be minimized for the efficient operation of the FCL, there must be a trade-off while designing the bias.

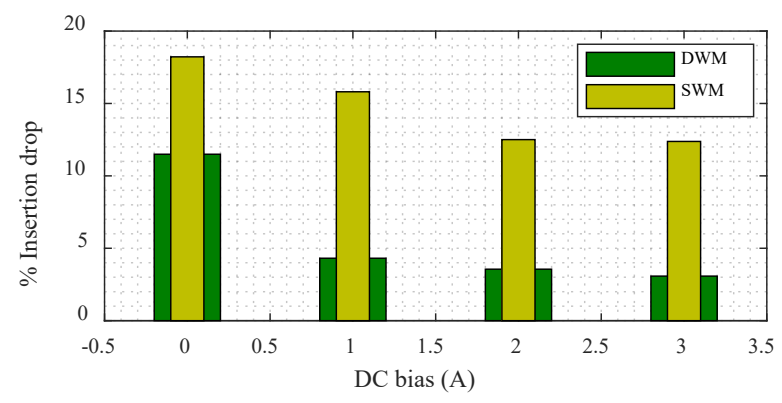

Figure 7. The insertion voltage drop

\subsection{The fault to pre-fault impedance ratio}

The fault to pre-fault impedance ratio is of critical importance in the current limiting process of the SCFCL. It is desired to have as large as possible the fault impedance under limiting state for effective current limitation, and the pre-fault impedance as small as possible for affecting the lowest possible voltage drop in the system. The ratio of fault to pre-fault impedance for both of the models is given in Table 3 . The comparative variations in the impedance ratio with bias are graphically plotted in Figure 8 .

It has been noted that both the pre-fault and fault impedance decreases with the increasing bias. However, the rate of reduction of pre-fault impedance dominates that of fault impedance, and hence the resulting ratio increases. The changes in the impedances with bias in the case of the single AC winding model were found to be marginal as against noticeable in the double AC winding model. However, the takeoff of a fault by the device is not smooth but associated with transients. The nature of the impedance during the pre-fault, fault, and transient conditions (zero degrees fault inception on voltage wave) is shown in Figure 9.

Table 3. The fault to pre-fault impedance ratio

\begin{tabular}{ccc}
\hline DC bias & $\begin{array}{c}\text { Double } \\
\text { AC winding model }\end{array}$ & $\begin{array}{c}\text { Single } \\
\text { AC winding model }\end{array}$ \\
\hline 0 & 0.49 & 1.00 \\
1 & 1.02 & 1.08 \\
2 & 1.10 & 1.20 \\
3 & 1.19 & 1.20 \\
\hline
\end{tabular}

Int J Elec \& Comp Eng, Vol. 11, No. 6, December 2021 : 4667 - 4677 


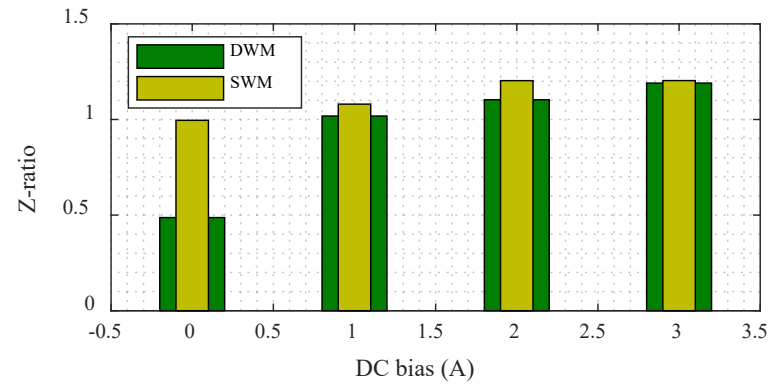

Figure 8. Fault to pre-fault impedance ratio

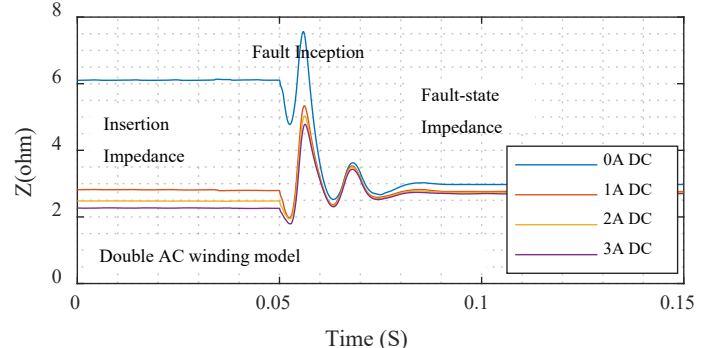

(a)

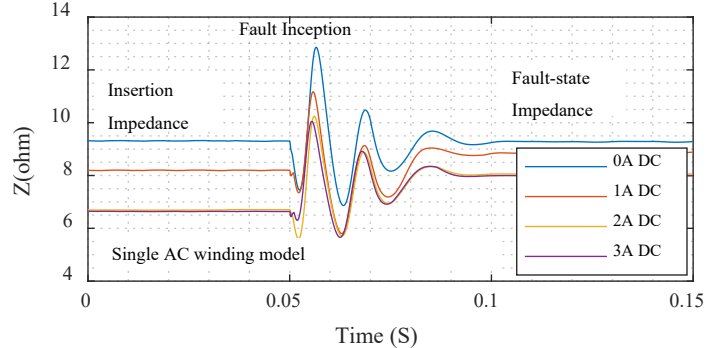

(b)

Figure 9. Impedance during the pre-fault, fault, and transient conditions; (a) double AC winding model, (b) single AC winding model

In the case of a double AC winding model, the unbiased operation has offered a significant steadystate impedance (above $6 \Omega$ ). The high impedance value had demonstrated the non-saturated core operation. For the bias of 1A and above, the steady-state impedance dropped to lower magnitudes and was decreasing as the bias level increases. The change above 1A DC bias is very marginal. The extensively high steady-state impedance values in the case of a single AC winding model had measured for all the designated DC bias. It also validates the more demand for the DC bias to force the core in the saturation region. The steady-state impedance affects the voltage drop contribution of the device and hence desired to be as minimum as possible. The higher values of the voltage drops in the single AC winding model are evident as recorded in Table 2. Also, as can be seen in Figure 7, the performance of the double AC winding model in terms of the voltage drop has been recorded much better compared with that of the single AC winding model.

The performance in terms of the asymmetry (transient) of the limited current waveform is also analyzed. The percentage rise from the steady-state peak to the maximum-peak of the controlled current has given in Table 4 . The higher values of the overshoots (DC component) in the short-circuit current handled by the single AC winding model have been noted. The DC component may be seen as an offset, and its magnitude is dependent upon the fault inception in addition to the system X/R ratio. It results in a higher instantaneous current than the steady-state short circuit current. The rated short-circuit breaking current is the highest value of current that the circuit breaker must be capable of breaking at its rated voltage. It is characterized by the rms value of its AC component and the aperiodic DC component. The level of the peak current before the interruption is the most significant parameter controlling the breaker's ability to clear faults. The longer and higher the dc offset value at the time of contact separation, the harder it will be for the breaker to interrupt the current. Hence, the fault control with the single AC winding model may credit a comparatively higher burden on the circuit breaker recruited for handing of the fault.

Table 4. Percentage rise from the steady-state peak to the maximum peak of limited current

\begin{tabular}{ccc}
\hline DC bias & $\begin{array}{c}\text { Double } \\
\text { AC winding model }\end{array}$ & $\begin{array}{c}\text { Single } \\
\text { AC winding model }\end{array}$ \\
\hline 0 & 12.85 & 27.43 \\
1 & 0.30 & 19.44 \\
2 & 0.21 & 18.13 \\
3 & 0.17 & 17.62 \\
\hline
\end{tabular}




\section{CONCLUSION}

It was evident from the magnetization characteristics that the injected DC bias controls the coil impedance, more the DC bias lower is the impedance to the AC coils. The magnetization curve plotted experimentally has validated the analytical calculations which, enabled the decision to select the bias levels. The 0A bias iron-core operation, although an unrealistic operating condition, was useful to compare and contrast the other applied biasing levels.

The two test models have exhibited the proof-of-the concept, though the performances under identical operating conditions are different. The current clipping of more than $65 \%$ had demonstrated by these test models. However, the voltage drop contributions in the case of the double AC winding model is well below the general statutory limits at and above the DC bias of 1A. For the single AC winding model, the voltage drop contributions are exceedingly high and are unacceptable. It reveals the need for comparatively more DC bias for a single AC winding model.

The performance in terms of current reduction, and the voltage drop, for the double AC winding model above 1 A DC bias, generated trivial improvements. It exposes that the under biasing of the core contributes to the higher voltage drop, and the over biasing, in addition to possible increased steady-state losses, may result in marginal performance improvements. Also, the fault to pre-fault impedance ratio decreases with the bias as the decrease in the pre-fault impedance (rate) is more with the bias. It is due to the shifting of the iron-core operating point towards the right on the saturation curve with the DC bias. And the larger component of the de-magnetizing AC force is compromised by the then applied bias.

The asymmetry in the transient impedance decreases with an increasing bias for both the models. The higher values of the overshoots (DC components) in the short-circuit current handled by the single AC winding model have been noted, which may recruit more burden on the subsequent circuit breaker taking the fault. The future work includes development of design methodology and its validation with the commercial design tool.

\section{ACKNOWLEDGEMENTS}

The authors are thankful to the Gokhale Education Society's R. H. Sapat College of Engineering, Management Studies and Research for providing laboratory facilities to carry out this research work.

\section{REFERENCES}

[1] S. C. Shekar, G. R. Kumar, and S. V. N. L. Lalitha, "A transient current based micro-grid connected power system protection scheme using wavelet approach," International Journal of Electrical and Computer Engineering (IJECE), vol. 9, no. 1, pp. 14-22, Feb. 2019, doi: 10.11591/ijece.v9i1.pp14-22.

[2] G. R. P. Kumar, D. Sattianadan, and K. Vijayakumar, "A survey on power management strategies of hybrid energy systems in microgrid," International Journal of Electrical and Computer Engineering (IJECE), vol. 10, no. 2, pp. 1667-1673, Apr. 2020, doi: 10.11591/ijece.v10i2.pp1667-1673.

[3] B. Banhthasit, C. Jamroen, and S. Dechanupaprittha, "Optimal Generation Scheduling of Power System for Maximum Renewable Energy Harvesting and Power Losses Minimization," International Journal of Electrical and Computer Engineering (IJECE), vol. 8, no. 4, pp. 1954-1966, Aug. 2018, doi: 10.11591/ijece.v8i4.pp1954-1966.

[4] M. Pathan, M. Al Owaifeer, M. Al Muhaini, and S. Z. Djokic, "Reliability Evaluation of Smart Distribution Grids with Renewable Energy Sources and Demand Side Management," Arab J Sci Eng, vol. 45, pp. 6347-6360, Mar. 2020, doi: 10.1007/s13369-020-04477-8.

[5] O. E. Oni, A. G. Swanson, and R. P. Carpanen, "Impact of LCC-HVDC multiterminal on generator rotor angle stability," International Journal of Electrical and Computer Engineering (IJECE), vol. 10, no. 1, pp. 22-34, Feb. 2020, doi: 10.11591/ijece.v10i1.pp22-34.

[6] M. Balasubbareddy, G. V. K. Murthy, and K. S. Kumar, "Performance evaluation of different structures of power system stabilizers," International Journal of Electrical and Computer Engineering (IJECE), vol. 11, no. 1, pp. 114 123, Feb. 202110.11591/ijece.v11i1.pp114-123.

[7] V. Lavanya and N. S. Kumar, "Control strategies for seamless transfer between the grid-connected and islanded modes of a microgrid system," International Journal of Electrical and Computer Engineering (IJECE), vol. 10, no. 5, pp. 4490-4506, Oct. 2020, doi: 10.11591/ijece.v10i5.pp4490-4506.

[8] H. Yang, Y. Zhang, Y. Ma, M. Zhou, and X. Yang, "Reliability evaluation of power systems in the presence of energy storage system as demand management resource," Int J Electr Power Energy Syst, vol. 110, pp. 1-10, Sep. 2019, doi: 10.1016/j.ijepes.2019.02.042.

[9] L. Kovalsky, X. Yuan, K. Tekletsadik, A. Keri, J. Bock, and F. Breuer, “Applications of Superconducting Fault Current Limiters in electric power transmission systems," IEEE Trans. Appl. Supercond., vol. 15, no. 2, pp. 2130-2133, Jun. 2005 doi: 10.1109/TASC.2005.849471.

[10] Y. Jia, Z. Shi, H. Zhu, L. Hao, J. Zou and J. Yuan, "Cognition on the Current-Limiting Effect of Saturated-Core Superconducting Fault Current Limiter," IEEE Transactions on Magnetics, vol. 51, no. 11, pp. 1-4, Nov. 2015, doi: 10.1109/TMAG.2015.2436715. 
[11] CIGRE, "Fault current limiters - Application, principles and testing," CIGRE working group A3.10, e-cigre.org., 2003.

[12] H. Radmanesh, S. H. Fathi, G. B. Gharehpetian, and A. Heidary, "Bridge type solid-state fault current limiter based on AC/DC reactor," IEEE Trans. Power Del., vol. 31, no. 1, pp. 200-209, Feb. 2016, doi: 10.1109/TPWRD.2015.2477106.

[13] B. Li, F. Guo, J. Wang, and C. Li, "Electromagnetic transient analysis of the saturated iron-core superconductor fault current limiter," IEEE Trans. Appl. Supercond., vol. 25, no. 3, pp. 1-5, Jun. 2015, doi: 10.1109/TASC.2014.2374191.

[14] N. Vilhena, P. Arsenio, J. Murta-Pina, A. Pronto, and A. Alvarez, "A methodology for modeling and simulation of saturated cores fault current limiters," IEEE Trans. Appl. Supercond., vol. 25, no. 3, pp. 1-4, Jun. 2015, doi: 10.1109/TASC.2014.2374179.

[15] A. Abramovitz, K. M. Smedley, F. De La Rose, and F. Moriconi, "Prototyping and testing of a $15 \mathrm{kV} / 1.2 \mathrm{kA}$ saturable core HTS fault current limiter," IEEE Trans. Power Del., vol. 28, no. 3, pp. 1271-1279, Jul. 2013, doi: 10.1109/TPWRD.2013.2256801

[16] J. X. Jin, S. X. Dou, H. K. Liu, and C. Grantham, "Preparation of high T/sub c/ superconducting coils for consideration of their use in a prototype fault current limiter," IEEE Trans. Appl. Superc., vol. 5, pp. 1051-1054, Jun. 1995, doi: 10.1109/77.402732.

[17] J. X. Jin et al., "Electrical application of a high T, superconducting saturable magnetic core fault current limiter," IEEE Trans. Appl. Superc., vol. 7, pp. 1009-1012, Jun. 1997, doi: 10.1109/77.614678.

[18] V. Keilin et al., "Model of HTS three-phase saturated core fault current limiter," IEEE Trans. Appl. Superc., vol. 10, pp. 2629-2630, Mar. 2000, doi: 10.1109/77.828361.

[19] B. P. Raju, K. S. Parton, and T. C. Bartram, "A current limiting device using superconducting d.c. bias. Application and prospects," IEEE Trans. Power Apparatus \& Systems, vol. 101, pp. 3173-3177, Sep. 1982, doi: 10.1109/TPAS.1982.317531.

[20] Y. Nikulshin, Y. Wolfus, A. Friedman, and Y. Yeshurun, "Improving the performance of saturated cores fault current limiters by varying winding density in the AC coils," IEEE Trans. Appl. Supercond., vol. 25, no. 3, pp. 1-5, Jun. 2015, doi: 10.1109/TASC.2014.2386323.

[21] Y. Nikulshin, A. Friedman, Y. Wolfus, V. Rozenshtein, and Y. Yeshurun, "Dynamic Desaturation Process in Saturated Cores Fault Current Limiters," IEEE Transactions on App. Supercond., vol. 22, no. 3, pp. 5601704-5601704, Jun. 2012, doi: 10.1109/TASC.2011.2179837 .

[22] M. Noe and M. Steurer, "High-temperature fault current limiters: Concepts, applications and development status," Supercond. Sci. Technol., vol. 20, no. 3, pp. 15-27, Mar. 2007.

[23] J. W. Moscrop, "Experimental analysis of the magnetic flux characteristics of saturated core fault current limiters," IEEE Trans. Magn., vol. 49, no. 2, pp. 874-882, Feb. 2013, doi: 10.1109/TMAG.2012.2214396.

[24] S. B. Abbott, D. A. Robinson, S. Perera, F. A. Darmann, C. J. Hawley, and T. P. Beales, "Simulation of HTS saturable core-type FCLs for MV distribution systems," IEEE Trans. Power Del., vol. 21, no. 2, pp. 1013-1018, Apr. 2006, doi:10.1109/TPWRD.2005.859300.

[25] S. Keyue and A. Alexander, "Development of Fault Current Controller Technology: Prototyping Laboratory Testing and Field Demonstration," University of California Irvine, California Energy Commission, 2011.

[26] V. Rozenshtein et al., "Saturated Cores FCL-A New Approach," IEEE Trans. on Appl. Supercond., vol. 17, no. 2, pp. 1756-1759, Jun. 2007, doi: 10.1109/TASC.2007.898153. 